\title{
TITLE:
}

\section{Carrier multiplication in carbon nanotubes studied by femtosecond pump-probe spectroscopy}

\section{$\operatorname{AUTHOR}(S)$ :}

Ueda, Akihiro; Matsuda, Kazunari; Tayagaki, Takeshi; Kanemitsu, Yoshihiko

\section{CITATION:}

Ueda, Akihiro ... [et al]. Carrier multiplication in carbon nanotubes studied by

femtosecond pump-probe spectroscopy. Applied Physics Letters 2008, 92(23): 233105.

ISSUE DATE:

2008-06

URL:

http://hdl.handle.net/2433/87355

RIGHT:

c 2008 American Institute of Physics. 


\title{
Carrier multiplication in carbon nanotubes studied by femtosecond pump-probe spectroscopy
}

\author{
Akihiro Ueda, Kazunari Matsuda, Takeshi Tayagaki, and Yoshihiko Kanemitsu ${ }^{\text {a) }}$ \\ Institute for Chemical Research, Kyoto University, Uji, Kyoto 611-0011, Japan
}

(Received 10 April 2008; accepted 21 May 2008; published online 10 June 2008)

\begin{abstract}
Carbon nanotubes are one of the excellent materials for studying the many-body effects of excitons because of their unique band structures and large exciton binding energies. We studied exciton population dynamics in single-walled carbon nanotubes using pump-probe transient absorption measurements. The temporal profiles of the transient absorption signals depend on the excitation intensity and excitation photon energy. We observe carrier multiplication in carbon nanotubes at room temperature, when the excitation photon energy exceeds the third subband exciton energy. (C) 2008 American Institute of Physics. [DOI: 10.1063/1.2943649]
\end{abstract}

Strongly confined electrons and excitons in lowdimensional semiconductor nanostructures, such as carbon nanotubes and nanocrystals, have unique electronic and optical properties compared with bulk semiconductors. ${ }^{1}$ Semiconductor carbon nanotubes have been studied extensively, both from the viewpoint of fundamental physics and due to their potential applications in electronics and photonics devices. ${ }^{2}$ A single-walled carbon nanotube (SWNT) about $1 \mathrm{~nm}$ in diameter with a length greater than several hundred nanometers is a prototype of one-dimensional (1D) structures. Strong Coulomb interactions in 1D structures lead to the formation of stable excitons with extremely large binding energies of 200-400 meV (Refs. 3 and 4) and enhance the many-body effects of excitons in SWNTs. For example, the quantized and fast Auger recombination of excitons and impact excitation by hot carriers have both been observed in SWNTs. ${ }^{5-10}$ In this letter, we report the observation of carrier multiplication $(\mathrm{CM})$ in SWNTs due to strong Coulomb interactions.

The CM process is the production of two or more electron-hole pairs by one high-energy photon well above the band gap energy. The relaxation of momentum conservation and strong Coulomb interactions between carriers in nanoscale space allow the observation of highly efficient CM phenomena in a variety of semiconductor nanocrystals. ${ }^{11-15}$ However, the detailed CM mechanism in semiconductor nanocrystals is controversial. ${ }^{16-19}$ Efficient $\mathrm{CM}$ has not been observed in systems other than nanocrystals. The achievement of efficient CM in semiconductors makes it possible to produce highly efficient solar cells with conversion efficiencies that exceed the Shockley-Queisser limit of $32 \% .^{20,21}$

Here, we examined CM in carbon nanotubes using femtosecond pump-probe spectroscopy. The temporal profiles of transient absorption signals depend on the excitation intensity and excitation photon energy. The dynamics of transient absorption is dominated by the Auger recombination process. A CM efficiency of 1.3 is determined from the transient absorption dynamics at room temperature when the photon energy exceeds the third intersubband exciton energy. The CM

\footnotetext{
${ }^{a)}$ Also at Photonics and Electronics Science and Engineering Center, Kyoto University, Kyoto 615-8510, Japan. Author to whom correspondence should be addressed. Electronic mail: kanemitu@scl.kyoto-u.ac.jp.
}

efficiency in carbon nanotubes is discussed in light of impact ionization.

The sample used in this work consisted of SWNTs in a surfactant suspension. SWNTs with a narrow chirality distribution were synthesized using a silica-supported Co-Mo catalyst (CoMoCAT) method (Southwest Nanotechnologies) ${ }^{22}$ following a procedure similar to that in Ref. 23. The SWNTs were dispersed in $\mathrm{D}_{2} \mathrm{O}$ with $1 \mathrm{wt} \%$ sodium dodecyl benzene sulfate by sonication with an ultrasonic processor for $0.5 \mathrm{~h}$. This suspension was then centrifuged for $2 \mathrm{~h}$ at $35000 \mathrm{~g}$.

The $150 \mathrm{fs}$ laser pulses from a Ti:sapphire regenerative amplifier system operating at $1 \mathrm{kHz}$ were used in the pumpprobe experiment. We used a probe pulse with a photon energy of $1.22 \mathrm{eV}$ from the optical parametric amplifier system. The photon energy of the probe pulse corresponds to the lowest exciton energy of $(6,5)$ carbon nanotubes. The pump pulse energies were $1.55,3.10$, and $4.65 \mathrm{eV}$. The polarizations of the pump and probe pulses paralleled each other in the experiments. All the measurements were carried out at room temperature.

Figure 1(a) shows the linear absorption spectrum of SWNTs in $\mathrm{D}_{2} \mathrm{O}$. The absorption peak observed at $1.22 \mathrm{eV}$ arises from the lowest exciton transition. The clear single absorption peak is due to the narrow chirality distribution of the CoMoCAT SWNTs. The energies of pump pulses (upward arrows) in this study are also shown in Fig. 1(a). Strong intersubband optical absorption occurs under a light electric
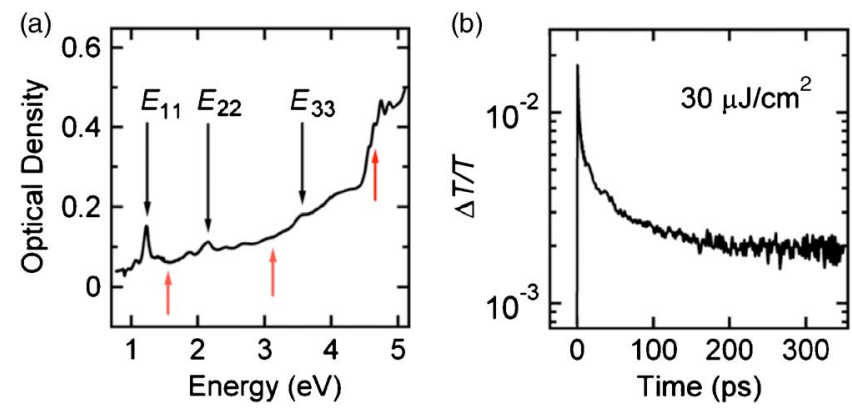

FIG. 1. (Color online) (a) Linear absorption spectrum of the SWNTs in $\mathrm{D}_{2} \mathrm{O}$. The downward arrow at $E_{11}$ indicates the energy of probe pulse corresponding to the lowest exciton energy of $(6,5)$ nanotubes. Upward arrows indicate the energies of pump pulses. (b) Transient absorption $(\Delta T / T)$ dynamics under $1.55 \mathrm{eV}$ excitation at $30 \mu \mathrm{J} / \mathrm{cm}^{2}$. 

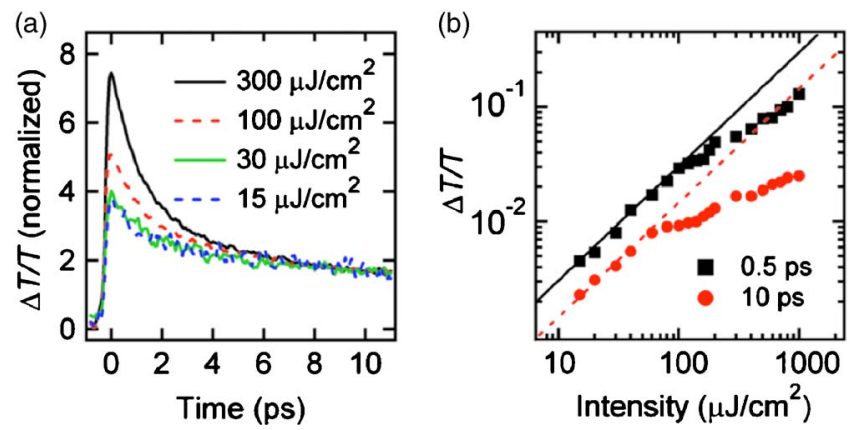

FIG. 2. (Color online) (a) Transient absorption $(\Delta T / T)$ dynamics under $1.55 \mathrm{eV}$ excitation at different intensities of $15,30,100$, and $300 \mu \mathrm{J} / \mathrm{cm}^{2}$. Transient absorption decays with various excitation intensities normalized by the long decay component ( $t=10 \mathrm{ps}$ ). (b) Excitation intensity dependence of the transient absorption signals at two delay times $(t=0.5$ and $10 \mathrm{ps})$. Black solid line and red dashed line show the linear dependences.

field parallel to the tube axis. This transition is due to optically allowed transitions between subbands with the same indices $i=j$. The exciton energy is described as $E_{i j}$, where the band structure is described by the valence subbands with index $i$ and conduction subbands with index $j$. As shown in Fig. 1(a), the three excitation energies of 1.55, 3.10, and $4.65 \mathrm{eV}$ exceed the exciton energies of $E_{11}, E_{22}$, and $E_{33}$, respectively. These laser pulses can produce electron-hole pairs in different subbands in $(6,5)$ carbon nanotubes.

Figure 1(b) shows the transient absorption decay excited by $1.55 \mathrm{eV}$ at an excitation density of $30 \mu \mathrm{J} / \mathrm{cm}^{2}$. It is clear that the temporal profile cannot be described by a single exponential function and that three exponential components (time constants of $\sim 1, \sim 30 \mathrm{ps}$, and $\sim 1 \mathrm{~ns}$ ) appear. The decay times of $\sim 30$ ps and $\sim 1$ ns are consistent with those obtained using transient photoluminescence spectroscopy. $^{24,25}$ These decays are determined by the trapping of excitons at defects and radiative recombination of excitons in SWNTs. ${ }^{24,25}$ Hereafter, we focus on the transient absorption changes of the fast-decay components on a picosecond time scale to clarify the $\mathrm{CM}$ mechanism and fast Auger recombination.

Figure 2(a) shows the excitation intensity dependence of the transient absorption decay from 15 to $300 \mu \mathrm{J} / \mathrm{cm}^{2}$ under $1.55 \mathrm{eV}$ excitation. The observed signals correspond to absorption bleaching due to excitons in SWNTs, as reported in pump-probe experiments. ${ }^{6,26}$ The decay profiles at longer delay times $(t>10 \mathrm{ps})$ do not depend on the excitation intensity. Then, all the data are normalized at 10 ps delay time. The normalized signal curves provide clear information about the excitation intensity-dependence of the exciton population dynamics. The fast-decay component $(t<5 \mathrm{ps})$ grows at increasing excitation intensity. The origin of this fast-decay will be discussed below.

Figure 2(b) summarizes the excitation intensity dependence of the transient absorption signals at two different delay times $(t=0.5$ and $10 \mathrm{ps})$. The magnitudes of the transient absorption signals at a $0.5 \mathrm{ps}$ delay time linearly increase with the excitation intensity up to $\sim 100 \mu \mathrm{J} / \mathrm{cm}^{2}$. By contrast, at a 10 ps delay time, saturation occurs at an excitation intensity of $50 \mu \mathrm{J} / \mathrm{cm}^{2}$. This indicates that, under intense excitation above $50 \mu \mathrm{J} / \mathrm{cm}^{2}$, the carrier density rapidly decreases on picosecond time scale.

Here, in order to understand the origin of the fast component in the transient absorption curves, we estimate the Downloaded 16 Nov 2009 to 130.54.110.32. Redistribution subject
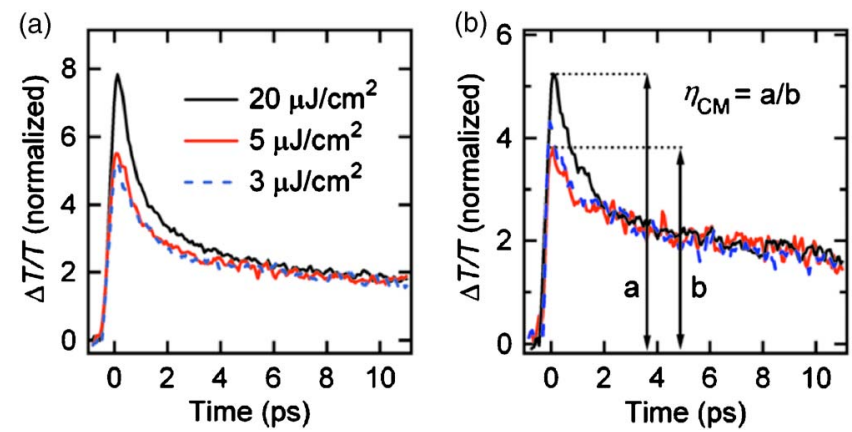

FIG. 3. (Color online) (a) Normalized transient absorption dynamics under $4.65 \mathrm{eV}$ excitation at different intensities of 3, 5, and $20 \mu \mathrm{J} / \mathrm{cm}^{2}$. (b) Normalized transient absorption decays under the weak excitation regime with different excitation photon energies (black solid line: $4.65 \mathrm{eV}$, blue broken line: $3.10 \mathrm{eV}$, and red line: $1.55 \mathrm{eV}$ ). This figure explains how to determine the $\mathrm{CM}$ efficiency, $\eta_{\mathrm{CM}}$, which is calculated from the ratio of the transient absorption signals at zero delay.

photoexcited electron-hole pair density in a SWNT. The saturation of the signal at $t=10 \mathrm{ps}$ begins at an excitation intensity of $50 \mu \mathrm{J} / \mathrm{cm}^{2}$ at $1.55 \mathrm{eV}$, corresponding to 2 $\times 10^{14}$ photon $/ \mathrm{cm}^{2}$. Absorption cross-section of approximately 550-nm-long carbon nanotubes (based on scanning electron microscopy observations) is estimated to be about $1 \times 10^{-14} \mathrm{~cm}^{2}$, according to Ref. 27 . Using these values, we determine that the average number of $e-h$ pairs (or excitons) in a carbon nanotube per pulse, $\left\langle N_{e x}\right\rangle$, is roughly equal to 2 at this excitation intensity. Therefore, at the intensity above $50 \mu \mathrm{J} / \mathrm{cm}^{2}$, we concluded that Auger recombination of excitons determine the transient absorption dynamics on several picoseconds. $^{5-7,26}$

Figure 3(a) shows the excitation intensity dependence of transient absorption dynamics under $4.65 \mathrm{eV}$ excitation. In the weak excitation regime where excitation intensity is less than about $10 \mu \mathrm{J} / \mathrm{cm}^{2}$, the decay dynamics is independent of the excitation intensity. Therefore, we conclude that the average absorbed photon number per a carbon nanotube is less than unity in these excitation intensities.

Figure 3(b) shows the transient absorption dynamics under a weak excitation density at photon energies of 1.55, 3.10 , and $4.65 \mathrm{eV}\left(15,10\right.$, and $3 \mu \mathrm{J} / \mathrm{cm}^{2}$, respectively). All of the decay curves are normalized for a long time delay $(t$ $=10 \mathrm{ps}$ ). We experimentally confirmed that in these weak intensity regions, the transient decay dynamics is independent of the excitation density. The fast-decay component due to Auger recombination does not appear in the decay curve under 1.55 and $3.10 \mathrm{eV}$ excitation. Note that the decay dynamics under $4.65 \mathrm{eV}$ excitation is faster than that under $1.55 \mathrm{eV}$ excitation, and that the lifetime of the fast-decay component under $4.65 \mathrm{eV}$ excitation is the same as the Auger recombination lifetime under intense $1.55 \mathrm{eV}$ excitation. This experimental result indicates that Auger recombination occurs under $4.65 \mathrm{eV}$ excitation, even in the weak intensity region. Since the signals of the Auger recombination process are a sign that more than two excitons are generated in a SWNT, we conclude that a single photon with an energy of $4.65 \mathrm{eV}$ produces two or more excitons under weak excitation conditions, that is, CM occurs in a SWNT.

We evaluate the CM quantum efficiency $\left(\eta_{\mathrm{CM}}\right)$, defined as the average number of excitons produced by one photon excitation. The $\Delta T / T$ signal amplitude is proportional to the number of excited excitons in the sample, since the transient to AIP license or copyright; see http://apl.aip.org/apl/copyright.jsp 
absorption change is very small. In the ensemble experiment, Schaller et al. demonstrated that the exciton density can be determined from signal amplitudes at zero delay in the time profiles normalized at a long delay time. ${ }^{28,29}$ In our case, the signal amplitudes at $10 \mathrm{ps}$ delay time are proportional to the number of excited carbon nanotubes, regardless of the excitation energies below and above the CM threshold. In Fig. 3 (b), the intensity ratio of $a / b$ at zero delay corresponds to $\eta_{\mathrm{CM}}$, where $a$ and $b$ are the amplitudes under 4.65 and $1.55 \mathrm{eV}$ excitation at zero delay, respectively. In our experiment, $\eta_{\mathrm{CM}}$ is estimated to be about 1.3 under $4.65 \mathrm{eV}$ excitation, while $\eta_{\mathrm{CM}}$ is almost 1 within the experimental error at $3.10 \mathrm{eV}$ excitation.

In bulk and nanostructured semiconductors, a possible mechanism of CM is the impact ionization. ${ }^{13,30}$ In impact ionization, the effective mass difference between holes and electrons is one of the most important factors determining the efficiency and threshold energy of CM. Due to the optical selection rule, the photoexcited electrons and holes have the same excess energies in carbon nanotubes. Since the effective masses of electrons and holes are the same, the threshold photon energy for $\mathrm{CM}, E_{\mathrm{CM}}$, is approximated by $E_{\mathrm{CM}}$ $\cong 3 E_{11} \cdot{ }^{13}$ This prediction is consistent with our experimental results. Therefore, $\mathrm{CM}$ in carbon nanotubes can be explained by impact ionization.

In conclusion, we report transient absorption and $\mathrm{CM}$ in carbon nanotubes using femtosecond pump-probe spectroscopy. The observed CM and quantum yields can be explained by impact ionization. We demonstrated that carbon nanotubes show unique optical responses because of their strong electron-electron interactions.

Part of this study was supported by a Grant-in-Aid for Scientific Research from the Japan Society for the Promotion of Science.

\footnotetext{
${ }^{1}$ See, for example, V. I. Klimov, J. Phys. Chem. 110, 16827 (2006).

${ }^{2}$ See, for example, S. V. Rotkin and S. Subramoney, Applied Physics of Carbon Nanotubes (Springer, Berlin, Heidelberg, New York, 2005).

${ }^{3}$ F. Wang, G. Dukovic, L. E. Brus, and T. F. Heinz, Science 308, 838 (2005).

${ }^{4}$ T. Ando, J. Phys. Soc. Jpn. 74, 777 (2005).
}

${ }^{5}$ F. Wang, G. Dukovic, E. Knoesel, L. E. Brus, and T. F. Heinz, Phys. Rev. B 70, 241403 (2004).

${ }^{6}$ L. Huang and T. D. Krauss, Phys. Rev. Lett. 96, 057407 (2006).

${ }^{7}$ K. Matsuda, T. Inoue, Y. Murakami, S. Maruyama, and Y. Kanemitsu, Phys. Rev. B 77, 033406 (2008).

${ }^{8}$ J. Chen, V. Perebeinos, M. Freitag, J. Tsang, Q. Fu, J. Liu, and P. Avouris, Science 310, 1171 (2005).

${ }^{9}$ L. Marty, E. Adam, L. Albert, R. Doyon, D. Ménard, and R. Martel, Phys. Rev. Lett. 96, 136803 (2006).

${ }^{10}$ V. Perebeinos and P. Avouris, Phys. Rev. B 74, 121410 (2006).

${ }^{11}$ R. D. Schaller and V. I. Klimov, Phys. Rev. Lett. 92, 186601 (2004).

${ }^{12}$ R. Ellingson, M. C. Beard, J. C. Johnson, P. Yu, O. I. Micic, A. J. Nozik, A. Shabaev, and A. L. Efros, Nano Lett. 5, 865 (2005).

${ }^{13}$ R. D. Schaller, M. A. Petruska, and V. I. Klimov, Appl. Phys. Lett. 87, 253102 (2005)

${ }^{14}$ M. C. Beard, K. P. Knutsen, P. Yu, J. M. Luther, Q. Song, W. K. Metzger, R. J. Ellingson, and A. J. Nozik, Nano Lett. 7, 2506 (2007).

${ }^{15}$ J. J. H. Pijpers, E. Hendry, M. T. W. Milder, R. Fanciulli, J. L. Herek, D. Vanmaekelbergh, S. Ruhman, D. Mocatta, D. Oron, A. Aharoni, U. Banin, and M. Bonn, J. Phys. Chem. C 111, 4146 (2007).

${ }^{16}$ A. Franceschetti, J. M. An, and A. Zunger, Nano Lett. 6, 2191 (2006).

${ }^{17}$ A. Shabaev, Al. L. Efros, and A. J. Nozik, Nano Lett. 6, 2856 (2006).

${ }^{18}$ G. Nair and M. G. Bawendi, Phys. Rev. B 76, 081304 (2007).

${ }^{19}$ M. Ben-Lulu, D. Mocatta, M. Bonn, U. Banin, and S. Ruhman, Nano Lett. 8, 1207 (2008).

${ }^{20}$ W. Shockley and H. J. Queisser, J. Appl. Phys. 32, 510 (1961).

${ }^{21}$ V. I. Klimov, Appl. Phys. Lett. 89, 123118 (2006).

${ }^{22}$ S. M. Bachilo, L. Balzano, J. E. Herrera, F. Pompeo, D. E. Resasco, and R. B. Weisman, J. Am. Chem. Soc. 125, 11186 (2003).

${ }^{23}$ M. J. O'Connell, S. M. Bachilo, C. B. Huffman, V. C. Moore, M. S. Strano, E. H. Haroz, K. L. Rialon, P. J. Boul, W. H. Noon, C. Kittrell, J. Ma, R. H. Hauge, R. B. Weisman, and R. E. Smalley, Science 297, 593 (2002).

${ }^{24}$ H. Hirori, K. Matsuda, Y. Miyauchi, S. Maruyama, and Y. Kanemitsu, Phys. Rev. Lett. 97, 257401 (2006).

${ }^{25}$ M. Jones, C. Engtrakul, W. K. Metzger, R. J. Ellingson, A. J. Nozik, M. J. Heben, and G. Rumbles, Phys. Rev. B 71, 115426 (2005).

${ }^{26}$ Y. Ma, L. Valkunas, S. L. Dexheimer, S. M. Bachilo, and G. R. Fleming, Phys. Rev. Lett. 94, 157402 (2005).

${ }^{27}$ L. J. Carlson, S. E. Maccagnano, M. Zheng, J. Silcox, and T. D. Krauss, Nano Lett. 7, 3698 (2007).

${ }^{28}$ R. D. Schaller, M. Sykora, S. Jeong, and V. I. Klimov, J. Phys. Chem. B 110, 25332 (2006).

${ }^{29}$ Even at weak intensity, the signal profile cannot be described by a single exponential decay, similar to the case of nanocrystals in Ref. 28. The excitation-intensity independent and fast decay is due to carrier trapping at defects.

${ }^{30} \mathrm{P}$. T. Landsberg, Recombination in Semiconductors (Cambridge University Press, Cambridge, 1991). 